\title{
A simple system for monitoring the timing of infant gaze to stimuli at specific locations
}

\author{
J. STEVEN REZNICK, KATARZYNA CHAWARSKA, STEPHANIE L. BETTS, and BRET LOGAN \\ Yale University, New Haven, Connecticut
}

\begin{abstract}
A system for monitoring the timing of infant gazes to specific locations is described. Infants sit on a parent's lap in a dark room and observe videotaped stimuli presented on one of four monitors. Infrared (IR) light sources provide illumination for an IR-sensitive video camera and are positioned so that the infant's direction of gaze can be readily apprehended on the basis of the reflections of the IR light sources relative to the infant's pupil. The parent monitors the infant's eyes through a headmounted display and keeps the infant in view of the video camera. Direction and timing of gaze shifts can be coded from frame-by-frame inspection of the videotape. We demonstrate that this coding is relatively accurate and that the present system provides some advantages over coding gaze direction on the basis of an unembellished video recording.
\end{abstract}

Development proceeds cephalocaudally (i.e., outward from the head). Thus, young infants control a limited set of responses, emitted primarily from the face. Researchers who study infants have developed techniques for exploiting crying, sucking, and eye movements to examine psychological development. Our goal in this paper is to describe a relatively simple system for monitoring a useful aspect of infant gaze: the timing and direction of the infant's gaze among stimuli at separate locations.

Human newborns make eye movements that are spatially coordinated with the locus of a sound (Butterworth \& Castillo, 1976; Wertheimer, 1961) and that track a slowly moving target (Kremenitzer, Vaughan, Kurtzberg, \& Dowling, 1979). ${ }^{1}$ Detailed analyses of infant eye movements during the 1 st year provide data that are relevant for theoretical accounts of the organization of visual activity (see, e.g., Bronson, 1990b, 1991, 1994, 1997; Hainline, Harris, \& Krinsky, 1990; Haith, 1980; Shea \& Aslin, 1990), the development of the object concept (see, e.g., Bremner, 1985; Moore, Borton, \& Darby, 1978), the formation of expectations (see, e.g., Haith, Wentworth, \& Canfield, 1993; Johnson, Posner, \& Rothbart, 1991, 1994), and the organization of neural development (see, e.g., Dean, Mayhew, \& Langdon, 1994; Johnson, 1990).

The earliest measurements of infant gaze were gathered through direct observation, but this crude technique was

We thank Olof Sigmarsdottir, Kathryn Sumberg, Jennifer Amsterlaw, and Yuan-Ying Hsu for assistance with data collection and coding; Bena Brandwein and J. J. Fueser for comments on early drafts of this manuscript; Kodak, Joe Szaszfai (Yale Audio Visual Center), William Murray (National Institute for Occupational Safety and Health), and Morris Waxler (Center for Device and Radiological Health) for expertise regarding the effects of infrared light; and the infants and parents who have generously contributed their time and energy to this research. Our research has been supported by Grant HD30678 from the U.S. Department of Health and Human Services, Public Health Service. Correspondence should be addressed to J. S. Reznick, Psychology Department, Yale University, Box 208205, New Haven, CT 06520-8205 (e-mail: steve.reznick@yale.edu). supplanted in the 1970s by electrooculography (EOG), in which small electrodes attached to the outer canthus of each orbit are used to detect eye movements. ${ }^{2}$ EOG allows sensitive detection of muscle contractions associated with eye movements, but calibration is required to determine the relation between eye movements and direction of gaze. This calibration can be problematic in infants because of their inability or unwillingness to detect or to follow target stimuli (Harris, Hainline, \& Abramov, 1981; Shupert \& Fuchs, 1988). Finocchio, Preston, and Fuchs (1990) have developed a technique that provides accurate EOG calibration relatively quickly, but other problems remain. For example, applying EOG electrodes to an infant can be slow and tedious, electrodes can cause discomfort in the infant or the parent, the electrodes can be vulnerable for infants who are capable of reaching and grasping, and eye movements that accompany head movements can be ambiguous (e.g., the infant may shift gaze to remain focused on a target).

An alternative approach for measuring eye movements is to photograph or film the eye and observe eye movements through subsequent analysis of these images. Kessen and Hershenson (1963) introduced the use of infrared (IR) illumination to eliminate the need for bright lights and to allow an observer to determine the infant's direction of gaze. In the Kessen and Hershenson procedure, IR illuminators were positioned beside each of two stimuli. A coder viewed the image of the infant's eye and determined which of the two points of light was nearer to the infant's pupil. In subsequent research using modifications of this technique, IR light sources were placed on the perimeter of a stimulus (e.g., at the vertices of a large triangle). The relation between the pupil and the reflections of the IR light was used to track the infant's scanning pattern (see, e.g., Haith, 1969; Maurer \& Salapatek, 1976; Salapatek, 1968; Salapatek \& Kessen, 1966).

Subsequent advances in technology led to the development of several systems for monitoring the infant's eye 
movements. ${ }^{3}$ Most of these systems use the bright-pupil/ corneal reflex technique, in which light from a small IR lamp is projected into the infant's eyeball coaxially with an IR-sensitive camera. The IR light is reflected from the retina. This back lights the pupil and makes it appear brighter than the surrounding eye. The IR light also reflects from the front surface of the eye, appearing as a small bright spot (i.e., the corneal reflex). The displacement of the bright spot from the center of the pupil is a function of the degree to which the optic axis of the eye is turned away from the camera location and thus indicates the direction of gaze.

The bright-pupil/corneal reflex technique has been particularly effective for infants 3 months old or younger who are willing to lie on their backs in a padded apparatus and observe stimuli on a monitor (see, e.g., Haith et al., 1993). Older infants are more comfortable seated upright in an infant seat or in proximity to an adult, which allows considerable range of head movement. In Hainline's (1981; Hainline \& Abramov, 1992) system, the infant is held on an experimenter's shoulder, facing backward. The experimenter faces forward and observes the infant's eyes in a monitor to keep the infant in camera range. Bronson (1993) described an apparatus in which a photo sensor uses the light from an IR light-emitting diode positioned on the infant's forehead to control a tracking mirror, to follow the infant's head movements, and thus to keep the infant's eye within camera range. Bronson reported that the system works reasonably well but that problems can arise for infants older than 2 months of age (e.g., tracking stops when infants turn away from the stimulus). Other problems also emerge for this type of recording system because of video distortion and corneal curvature (Bronson, 1983), but these can sometimes be corrected (see, e.g., Bronson, 1990a; Harris et al., 1981).

Our pilot studies indicated that infants $4-12$ months of age would be most cooperative and attentive if seated on a parent's lap with relative freedom of movement. Direction of gaze has been coded successfully in this configuration with unembellished videotaped recordings of the infant's eyes and face (see, e.g., DiLalla et al., 1990; Jacobson et al., 1992; Reznick, 1994). However, this configuration introduces several problems: (1) It is difficult to maintain an image of the infant's eyes in the video camera; (2) the direction of gaze is not always apparent; (3) infants may be distracted by irrelevant aspects of the apparatus and the testing room; and (4) parents may deliberately or unintentionally influence the infant's direction of gaze. We also wanted to use videotaped stimuli rather than computergenerated stimuli to make the visual experience as salient as possible and to provide flexibility in the location and size of the stimulus presentation. Finally, we sought a technique for recording eye movements that would be relatively simple and inexpensive, would capitalize on recent advances in video recording technology, and would provide the appropriate level of information (i.e., we are interested in the timing of infant gaze shifts away from the stimulus at one location and toward the stimulus at another location rather than the detailed pattern of gaze shifts that the infant uses to inspect the stimulus at a particular location).

The system described here solves these problems and allows accurate assessment of the timing of gaze toward stimuli at various locations in infants of 4-12 months. Infants sit on a parent's lap in a dark room and observe short presentations of videotaped stimuli that form a pattern or a random sequence among four separate video monitors. IR light sources provide illumination for an IR-sensitive video camera and are positioned so that the direction and timing of gaze shifts to the four locations can be readily apprehended on the basis of the reflections of the IR light sources relative to the infant's pupil. The parent monitors the infant's eyes through a head-mounted display to keep the infant in view of the video camera. The timing of gaze shifts is coded from frame-by-frame inspection of the videotape.

\section{APPARATUS}

IR light is provided by four $5 \frac{1}{2}$-in. safelights (Calumet BR-9000) each containing a single $40-\mathrm{W}$ bulb and filtered through a Kodak No. 11 IR filter. The advantage of a safelight, which is normally for darkroom use, is that the sealed fixture emits no visible light. An additional advantage is that safelights are relatively inexpensive. The disadvantages of this configuration are that the sealed fixture can become hot and the IR filter disguises the fact that the bulb is on. We connect the safelights to a timer-controlled power strip to prevent inadvertent failures to extinguish them. As shown in Figure 1, each safelight is mounted to the outside of a monitor, oriented toward the infant. The rim of the safelight is 5 in. from the edge of the monitor.

The lack of visible light makes self-protection from radiation a concern. Injury thresholds have not been established for exposure to IR light in children, but there is no biological or biophysical reason to expect that the threshold for IR damage is significantly different for the eyes of infants and the eyes of adults (Pitts \& Kleinstein, 1993; Waxler, 1996, personal communication). Threshold limit values for exposure of the human eye to IR light are very high (American Conference of Governmental Industrial Hygienists, 1996; Waxler \& Hitchins, 1986). The lamp intensity and exposure duration in the present configuration are trivial compared with IR light in occupational contexts and with natural sources (e.g., sunlight). Thus we are confident that the apparatus described in this paper poses no risk for infants or their parents.

The centers of the 13-in. monitors are 28 in. apart on the horizontal and vertical dimensions. Audio signals from each stimulus can be played simultaneously through all four monitors or through just the monitor that is active, thereby adding an auditory cue for stimulus location. For some applications, it is necessary to have the monitors completely invisible in the darkness. However, if infants are expected to make anticipatory gaze shifts before a stimulus appears, we have found it useful to provide a dim border of lights that demarcates each screen. We accom- 


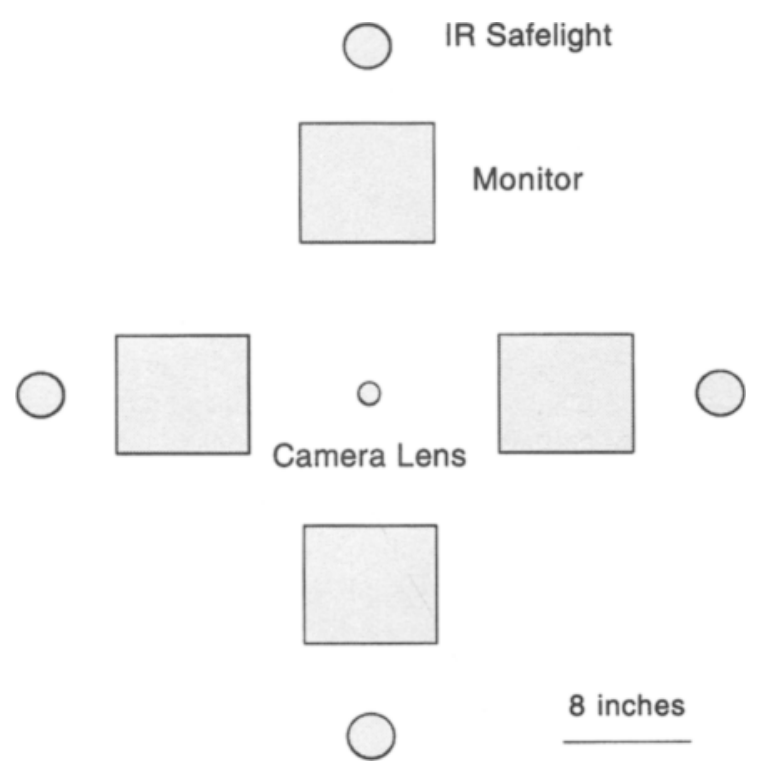

Figure 1. Configuration of apparatus.

plished this by using a frame composed of 50 small bulbs, mounted 1 in. apart and powered by a variable voltage supply set at $40 \mathrm{~V}$. The dimly illuminated frame helps infants locate the various sites where an upcoming stimulus may appear. Because all possible locations are framed, the frames provide no cue as to which location will be active.

An image of the infant's eyes is recorded using an IRsensitive, charge-coupled device camera with spectral sensitivity of 400-1,300 nm (Watec WAT-209, part 85291, FJW Optical Systems, Inc., Palatine, IL) located in the center of the apparatus. A 48-mm lens allows acceptable resolution of the eyes and much of the face at a distance of $50 \mathrm{in}$. The image of the face is recorded on a videocassette recorder (VCR) along with the signal from a Panasonic WJ-810 date/time generator. This signal contains the date, a video stopwatch running at increments of $10 \mathrm{msec}$, and an alarm code that can be controlled by the computer and used to indicate the onset, offset, and location of each stimulus. The recorded image also contains an insert screen with a view of the apparatus from a camera located behind the infant. The insert allows visual inspection of the timing, location, and identity of each stimulus presentation.

The parent wears a head-mounted display (Virtual IGlass Personal Display System, Virtual I/O, Seattle, WA) that provides the parent with the image of the infant's face that is being recorded on the VCR. The parent is instructed to position the infant's body so that the infant's eyes remain in view. The head-mounted display might be distracting for some infants, but because the testing room is dark and the parent can behave naturally (i.e., adjust the infant's seating position), infants do not realize that the head-mounted display is present. The back of the headmounted display is blocked so that the parent sees only the video image. Earphones on the head-mounted display provide ambient sound from the room. Thus, the parent does not know which location contains the active stimulus. We usually ask the parent to hold the infant on his/her lap, which is a position that most infants seem to prefer. However, if the height of the apparatus can be adjusted easily, infants can also be held looking backward over the parent's shoulder, as in Hainline's (Hainline, 1981; Hainline \& Abramov, 1992) system.

Each infant sees a sequence of stimulus presentations (e.g., a recording of a mechanical monkey that claps a pair of cymbals). A typical experiment starts with a stimulus presentation on the left monitor for $1 \mathrm{sec}$, followed by a $1-\mathrm{sec}$ intertrial interval (ITI), and then a stimulus presentation on the right monitor for $1 \mathrm{sec}$, followed by a 1 -sec ITI. This pattern repeats until the infant has seen 40 stimulus presentations. In another typical experiment, the stimulus presentation at one location is constant, with intermittent but temporally predictable stimulus presentations at some other location.

\section{INTERPRETING THE VIDEO RECORD}

We are primarily interested in determining when infants leave each stimulus location relative to the stimulus onset at the next location in the sequence. Trials on which the infant leaves a location before the onset of the next stimulus (or very quickly after stimulus onset) suggest that the infant has anticipated the upcoming stimulus. Trials on which the infant leaves a location after the onset of the next stimulus suggest that the gaze shift is a reaction to the new stimulus. In either case (i.e., for anticipations or for reactions), we are also interested in determining the accuracy of the initial saccade and the location that the infant ultimately fixates.

Some trials are lost because the infant's eyes are not in view. In a recent experiment, 12 infants between 6 and 12 months old saw three 40-trial episodes, with complexity of the sequence increasing across episodes. Gaze could be coded for more than $90 \%$ of trials on the first episode and more than $80 \%$ of trials on the second and third episodes. The decline in codable trials reflects infant fatigue and agitation but represents a relatively minimal data loss for cognitive assessments of infants in this age range.

Detecting gaze shifts is facilitated by reflections from the four IR safelights. When an infant gazes toward the center of the apparatus, the reflections of the safelights can be seen in the videotaped image as dots of light at the edges of both pupils, as shown in the center image in Figure 2. When the infant's gaze shifts toward one of the monitors, the position of one or more dots changes relative to the pupil. For example, if the infant gazes toward the monitor on the left, the dot that was on the outside of the left pupil moves to the center of the pupil (relative to the other dots). This effect can be seen in the left image in Figure 2. A parallel effect occurs when the infant gazes toward the monitor on the right, as seen in the right image in Figure 2. Finally, a gaze shift toward the monitor that is above or 

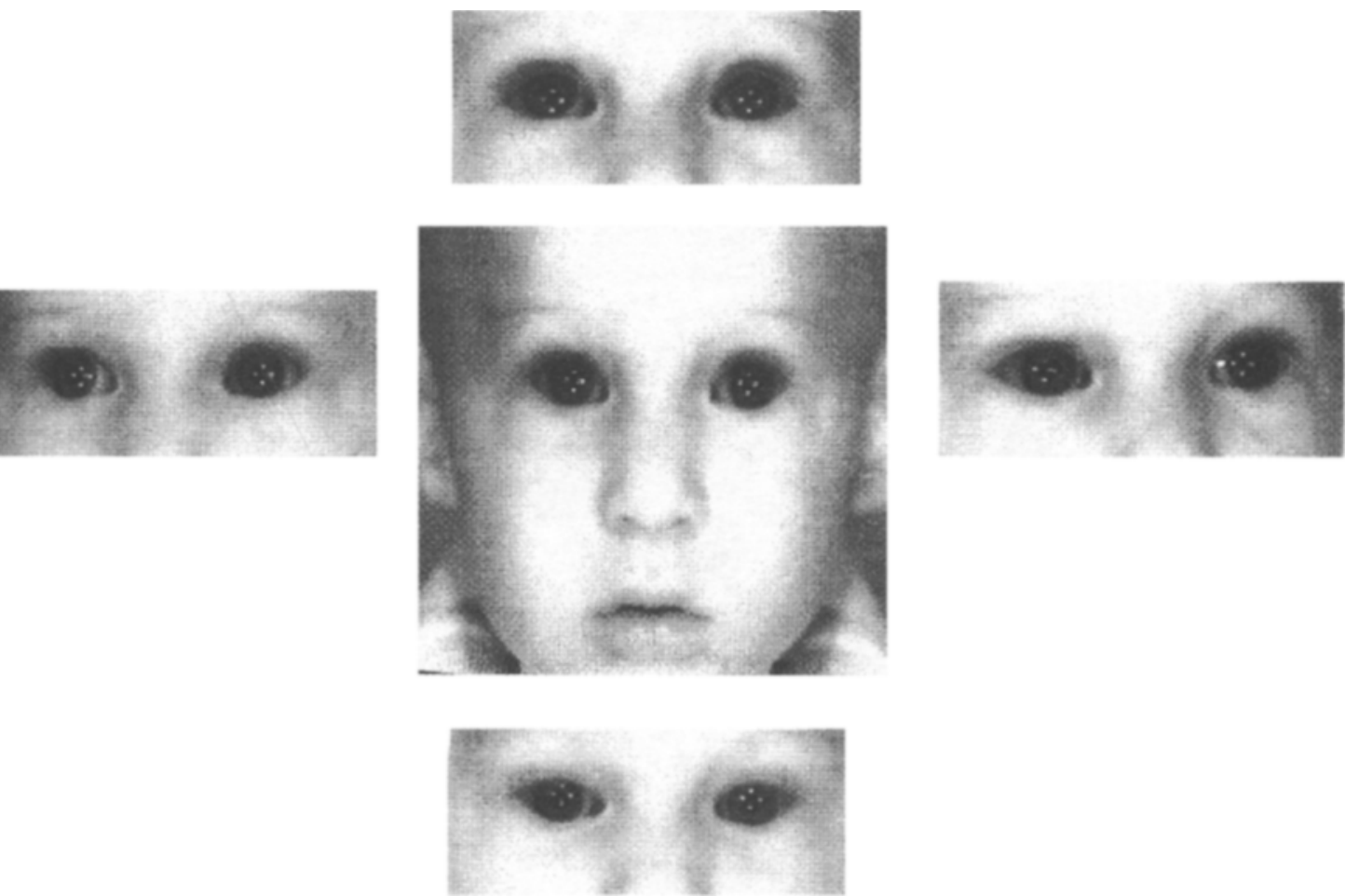

Figure 2. Videotaped image of a 9-month-old infant seated 50 in. away from the apparatus, looking to the center, and to stimuli presented to the left, right, above, and below center.

below the center of the apparatus causes the dots above or below each pupil to shift toward the center of the pupil, as shown in the upper and lower images in Figure 2.

To quantify the timing of the first gaze shift on each trial, we scan the videotape of the infant's eyes at a slow speed until the dot at the center of either pupil shifts toward the edge of the pupil. The IR dot facilitates coding because it establishes a specific criterion for identifying the first gaze shift. Subsequent frame-by-frame views of the eye before and after the shift are used to isolate the specific frame in which the shift begins. The time on the video stopwatch associated with this frame is recorded as the time of the gaze shift. The playback rate on the Panasonic AG-1960 VCR limits the accuracy of this assessment to $16-20 \mathrm{msec}$. The VCR is shifted to a slow speed again so that we can determine the accuracy of the initial saccade (i.e., orthometric, hypometric, or hypermetric) and the overall direction of the gaze shift (i.e., it is usually to the active or to-be-active target, but in some circumstances it is to a previously active target or to an irrelevant location). The coding of direction of gaze can be determined on the basis of the change in dot pattern (i.e., the top, bottom, left, or right dot will be in the center of the pupil). When the direction of gaze shift is determined, the target of the gaze shift is recorded and we begin scanning again for the next gaze shift away from what is now considered the previous target.

\section{RELIABILITY OF CODING}

We have no technique for calibrating the absolute accuracy of gaze shifts, but the pattern of response over trials suggests that infants are indeed shifting their gaze between the locations of relevant targets on most trials. We attempted to quantify the validity of coding the direction of gaze by preparing a videotape of an adult subject who shifted her gaze among the four locations according to a predetermined pattern for 30 trials. A coder who was not aware of the pattern attempted to determine the direction of gaze on each trial and was correct on $100 \%$ of the 30 trials.

We used a similar strategy for quantifying the accuracy with which initial gaze shifts could be designated as orthometric, hypometric, or hypermetric. An adult subject made 16 orthometric, 15 hypometric, and 10 hypermetric gaze shifts in a random order according to a predetermined script. A coder who was not aware of the pattern viewed the videotape and correctly identified the designation of 39 of the 41 gaze shifts $(95 \%)$.

The reliability for coding the timing of gaze shifts can be established by having an independent second observer code the videotape. Reliability tends to be strong. For example, in a study in which 6-, 9-, and 12-month-old infants saw alternating targets (as described earlier), a second observer recoded 6 randomly selected infants, 2 at each age. The comparison of two independent assessments indi- 
cated agreement within \pm 1 frame (i.e., $20 \mathrm{msec}$ ) on $84 \%$ of trials, and \pm 2 frames (i.e., $40 \mathrm{msec}$ ) on $90 \%$ of trials. Agreement on direction of gaze shift approached $100 \%$.

Finally, we compared the efficacy of this system with coding the timing of gaze shifts from an unembellished videotape (i.e., without IR light). Eight infants between 6 and 12 months of age saw two presentations in which 30 stimulus events appeared on one of two framed monitors. For half of the infants, the pattern for both presentations was symmetric: The other half saw an equal number of events at each location, but the sequence of locations was random. Each infant saw two presentations that were identical except that the room was dark and the IR lights were illuminated for one presentation. The room was lit and the IR lights were not illuminated for the other presentation.

Two independent coders recorded time and direction of gaze shift for each infant on each condition (i.e., with IR light and without IR light). The most obvious difference between conditions was a significant increase in the percentage of trials in which infants made a gaze shift that was on target (i.e., to the left or the right) instead of off target (i.e., anywhere other than one of the appropriate locations). An analysis of variance with coder and condition as within-subject effects revealed a main effect of condition $[F(1,7)=13.43, p<.01]$ but no other significant effects or interactions. The percentage of on-target shifts in the dark (i.e., with IR light) was $91 \%$, compared with $69 \%$ ontarget shifts in the light (i.e., without IR light). This indicates that one advantage of this system is that it makes it possible to test infants in the dark and thus to eliminate much of the distraction that is evoked by the apparatus and the room in which it is located.

We compared the two coders' estimates of when the infant's gaze shifted for the IR light and no-IR light conditions and found that agreement was relatively high in both: agreement within \pm 1 frame (i.e., $20 \mathrm{msec}$ ) on $95 \%$ of trials with IR light and on $92 \%$ of trials without IR light. Both coders were highly skilled and probably approached the ceiling of accuracy possible for videotaped recordings of infant direction of gaze. Coding on the basis of the IR signal was reported to be easier and faster. We suspect that a greater difference between conditions would emerge for observers who have had less experience coding infant gaze.

\section{DISCUSSION}

The system described here offers some advantage over other techniques for assessing infant eye movements. In comparison with the use of unembellished videotapes for coding gaze timing and direction, the IR light makes it possible to test infants in a dark or extremely dim room, thus avoiding distractions that can invalidate a large percentage of trials. Although the IR reflection did not improve the reliability between two experienced coders' assessment of gaze shift timing, it did make the videotaped image easier to interpret. Specifically, the direction of each gaze shift is revealed by the position of the pupil relative to the four IR dots, and the onset of the initial gaze shift can be referenced to the frame in which a dot at the center of the pupil shifts toward the edge of the pupil. Finally, the system described here has the advantage of precluding the parent's influence on the infant's direction of gaze because the head-mounted display prevents the parent from determining where an active stimulus is appearing. It seems unlikely that subtle cues from the parent would have much effect on the infant's direction of gaze, but this is still a potential flaw whenever the infant is seated on the parent's lap.

In comparison with sophisticated systems for monitoring direction of infant gaze (see, e.g., Bronson, 1993), the system described here has the advantage of requiring simpler and less expensive equipment and of allowing the infant more freedom of movement. However, this system does not allow the accuracy that is needed for some applications. Specifically, it cannot be used to measure scanning patterns (i.e., the direction and duration of fixations during the inspection of a stimulus). In sum, the present technique seems particularly applicable to somewhat older infants, to procedures that can and should be conducted in a darkened room, and to research in which the pattern and timing of selection among alternative stimulus locations provide sufficient data.

\section{REFERENCES}

american Conference of Governmental Industrial Hygienists (1996). Threshold limit values and biological exposure indices for 1995-96. (Available from ACGIH, 6500 Glenway Ave., Bldg. D-7, Cincinnati, $\mathrm{OH} 45211$ )

BREMNER, J. G. (1985). Object tracking and search in infancy: A review of data and a theoretical evaluation. Developmental Review, 5, 371-396.

Bronson, G. W. (1983). Potential sources of error when applying a corneal reflex eye-monitoring technique to infant subjects. Behavior Research Methods \& Instrumentation, 15, 22-28.

BRONSON, G. W. (1990a). The accurate calibration of infants' scanning records. Journal of Experimental Child Psychology, 49, 79-100.

Bronson, G. W. (1990b). Changes in infants' visual scanning across the 2- to 14-week age period. Journal of Experimental Child Psychology, 49, 101-125.

BRONSON, G. W. (1991). Infant differences in rate of visual encoding. Child Development, 62, 44-54.

Bronson, G. W. (1993). An eye monitoring system for infant subjects. Behavior Research Methods, Instruments, \& Computers, 25, 464-467

BRONSON, G. W. (1994). Infants' transitions toward adult-like scanning. Child Development, 65, 1243-1261.

Bronson, G. W. (1997). The growth of visual capacity: Evidence from infant scanning patterns. In C. Rovee-Collier \& L. P. Lipsitt (Eds.), Advances in infancy research (Vol. 11, pp. 109-141). Norwood, NJ: Ablex.

Butterworth, G., \& Castilla, M. (1976). Coordination of auditory and visual space in newborn human infants. Perception, 5, 155-160

Dean, P., Mayhew, J. E. W., \& Langdon, P. (1994). Learning and maintaining saccadic accuracy: A model of brainstem-cerebellar interactions, Journal of Cognitive Neuroscience, 6, 117-138.

Dilalla, L. F., Thompson, L. A., Plomin, R., Phillips, K., Fagan, J. F., Haith, M. M., Cyphers, L. H., \& FulkeR, D. W. ( 1990). Infant predictors of preschool and adult IQ: A study of infant twins and their parents. Developmental Psychology, 26, 759-769.

Finocchio, D. V., Preston, K. L., \& Fuchs, A. F. (1990). Obtaining a quantitative measure of eye movements in human infants: A method of calibrating the electrooculogram. Vision Research, 30, $1119-1128$.

HAINLINE, L. (1981). An automated eye movement recording system for use with human infants. Behavior Research Methods \& Instrumentation, 13. 20-24. 
Hainline, L., \& Abramov, I. (1992). Assessing visual development: Is infant vision good enough. In C. Rovee-Collier \& L. P. Lipsitt (Eds.), Advances in infancy research (Vol. 7, pp. 39-102). Norwood, NJ: Ablex.

Hainline, L., Harris, C. M., \& Krinsky, S. (1990). Variability of refixation in infants. Infant Behavior \& Development, 13, 321-342.

Haiтh, M. M. (1969). Infrared television recording and measurement of ocular behavior in the human infant. American Psychologist, 24, 279-283.

Haith, M. M. (1980). Rules that babies look by. Hillsdale, NJ: Erlbaum.

Haith, M. M., Wentworth, N., \& Canfield, R. L. (1993). The formation of expectation in early infancy. In C. Rovee-Collier \& L. P. Lipsitt (Eds.), Advances in infancy research (Vol. 8, pp. 251-297). Norwood, NJ: Ablex.

Harris, C. M., Hainline, L., \& Abramov, I. (1981). A method for calibrating an eye-monitoring system for use with infants. Behavior Research Methods \& Instrumentation, 13, 11-17.

Jacobson, S. W., Jacobson, J. L., O'Neill, J. M., Padgett, R. J., Frankowski, J. J., \& BiHUn, J. T. (1992). Visual expectation and dimensions of infant information processing. Child Development, 63, $711-724$.

Johnsun, M. H. (1990). Cortical maturation and the development of visual attention in early infancy. Journal of Cognitive Neuroscience, 2, $81-95$.

Johnson, M. H., Posner, M. I., \& Rothbart, M. K. (1991). Components of visual orienting in early infancy: Contingency learning, anticipatory looking, and disengaging. Journal of Cognitive Neuroscience, 3, 335-344.

Johnson, M. H., Posner, M. I., \& Rothbart, M. K. (1994). Facilitation of saccades toward a covertly attended location in early infancy. Psychological Science, 5, 90-93.

Kessen, W., \& Hershenson, M. (1963, August). Ocular orientation in the human newborn infant. Paper presented at the annual meeting of the American Psychological Association.

Kremenitzer, J. P., Vaughan, H. G., Jr., Kurtzberg, D., \& DowlING, K. (1979). Smooth-pursuit eye movements in the newborn infant. Child Development, 50, 442-448.

MAurer, D. (1975). Infant visual perception: Methods of study. In L. B.
Cohen \& P. Salapatek (Eds.), Infant perception: From sensation to cognition: Vol. I. Basic visual processes (pp. 1-76). New York: Academic Press.

Maurer, D., \& Salapatek, P. (1976). Developmental changes in the scanning of faces by young infants. Child Development, 47, 523-527.

MoORE, M. K., BorTON, R., \& DARBY, B. L. (1978). Visual tracking in young infants: Evidence for object identity or object permanence? Journal of Experimental Child Psychology, 25, 183-198.

PitTs, D. G., \& Kleinstein, R. N. (1993). Environmental vision: Interactions of the eye, vision, and the environment. Boston: ButterworthHeinemann.

REZNICK, J. S. (1994). In search of infant expectation. In M. M. Haith, J. B. Benson, \& R. J. Roberts, Jr. (Eds.), The development of futureoriented processes (pp. 39-59). Chicago: University of Chicago Press.

SALAPATEK, P. (1968). Visual scanning of geometric figures by the human newborn. Journal of Comparative \& Physiological Psychology, 66, 247-258.

SALAPATEK, P., \& KESSEN, W. (1966). Visual scanning of triangles by the human newborn. Journal of Experimental Child Psychology, 3, 155-167.

SHEA, S. L., \& AsLin, R. N. (1990). Oculomotor responses to step-ramp targets by young human infants. Vision Research, 30, 1077-1092.

SHUPERT, C., \& FUCHS, A. F. (1988). Development of conjugate human eye movements. Vision Research, 28, 585-596.

WAXLER, M., \& Hitchins, V. M. (1986). Optical radiation and visual health. Boca Raton, FL: CRC Press.

WERTHEIMER, M. (1961). Psychomotor coordination of auditory and visual space at birth. Science, 134, 1692

\section{NOTES}

1. See Hainline and Abramov (1992) for a review of infant visual development.

2. See Maurer (1975) for a review of EOG data.

3. See Bronson (1993) for a review of eye movement systems.

(Manuscript received May 28, 1996; revision accepted for publication December 17, 1996.) 